\section{Indicadores de fragilidad en adultos mayores del sistema público de salud de la ciudad de Antofagasta}

\author{
CATALINA TAPIA P., ${ }^{1, a}$, YENNY VALDIVIA-ROJAS ${ }^{1, b}$, \\ HÉCTOR VARELA V. ${ }^{2, \mathrm{c}}$, ANDRÉS CARMONA G. ${ }^{1, \mathrm{~d}}$, \\ VERÓNICA ITURRA M. ${ }^{1, \mathrm{e}}$, MÓNICA JORQUERA C. ${ }^{1, \mathrm{f}}$
}

\section{Rates of frailty among older people ascribed to chilean primary care clinics}

Background: Frailty is not universal among older people but increases the risk of dependence. Aim: To assess frailty among older people and its relationship with biological, psychological and social factors. Material and Methods: Seven hundred fifty four older people aged $73 \pm 6$ years (61\% females), attending a public primary care were assessed. Frailty was defined according to Fried criteria that considers inexplicable weight loss, tiredness, muscle weakness and lack of physical activity. Results: Absence of frailty, pre-frailty and frailty was found in 26, 69 and 5\% of participants, respectively. Significant differences between frailty groups were observed for age, gender, years of studies, minimental and self-efficacy scores. Among participants defined as being in a pre-frail condition, $59 \%$ were non-disabled without risk and $41 \%$ non-disabled in risk, according to the functional assessment for older people used in Chilean primary care clinics. Conclusions: Frailty among older people is associated with increasing age, education, cognitive status and self-efficacy.

(Rev Med Chile 2015; 143: 459-466)

Key words: Aged, Frail Elderly, Primary health care.

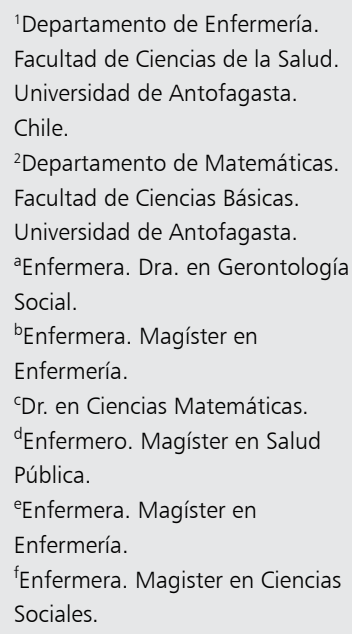

Recibido el 12 de agosto de 2013, aceptado el 26 enero de 2015

Correspondencia a:

Dra. Catalina Tapia Pinto Departamento de Enfermería Universidad de Antofagasta Fono: 055-2-637422

catalina.tapia@uantof.cl
E n Chile, según censo 2012, 14\% de la población chilena tiene más de 60 años ${ }^{1}$; según proyecciones del INE el 2015 las personas sobre los 75 años de edad pueden alcanzar el $4 \%$ de la población total ${ }^{2}$. Este aumento de edad implica discapacidad. La población total con una o más discapacidad alcanza los 2.119.316, para el rango de 45 a 59 años, la cifra es de 530.088 y desde los 60 y más años aumenta a 889.720 (41,98\%). La dificultad de movimiento en el mismo rango etario alcanza el 53,86\% ${ }^{1}$. Los adultos mayores (AM) pueden tener alguna discapacidad, que puede asociarse con la dependencia; y ambas se relacionan al fenómeno de fragilidad. En el estudio de dependencia en AM chilenos (EDPM 2009) ${ }^{3}$ se encontró que la prevalencia aumenta con la edad llegando a 52,9\% en los mayores de 80-84 años, valor que difiere según el sexo, $45,0 \%$ para los hombres y 57,0\% para las mujeres; considerando que sobre los 80 años existen más mujeres, es razonable esperar niveles de dependencia asociada a discapacidad y niveles de fragilidad.

La fragilidad no es el estado previo a la discapacidad sino la predisposición a desarrollarla, y puede devenir desde la autonomía o desde la dependencia ${ }^{4}$. Esto implica el riesgo que un AM autónomo y/o autovalente pueda como consecuencia de una enfermedad u otro evento, volverse frágil. La fragilidad es un problema de salud asociado a personas de edad muy avanzada, recibe diferentes definiciones como un síndrome geriátrico, un estado o una condición, todas incorporan los siguientes elementos: disminución o falla en la reserva funcional, se compromete la respuesta individual frente a cualquier tipo de estrés, lleva al deterioro o menoscabo del estado 
de salud, y puede significar la institucionalización, la hospitalización e incluso la muerte ${ }^{5,6}$.

La definición de fragilidad de Linda Fried et $\mathrm{al}^{7}$, corresponde a un síndrome y plantea la idea de un fenotipo de fragilidad, caracterizado por pérdida de peso inexplicable, cansancio, debilidad muscular, marcha lenta y poca actividad física.

En Chile, desde el 2004 se aplica la evaluación funcional del AM (EFAM) al sector público, (FONASA) que corresponde a 76,2\% de los beneficiarios del sistema de salud chileno ${ }^{8}$ y a contar de 2006 al sector privado. Esta evaluación es un predictor de pérdida de funcionalidad del AM, que consta de la sección A donde clasifica dependencia o autovalencia y la sección B que discrimina la presencia de riesgos entre los autovalentes ${ }^{9}$. A través de una guía clínica se recomendó ${ }^{10}$ a los equipos de atención primaria de salud realizar además del EFAM, acciones preventivas para pesquisa de factores de riesgo que puedan generar fragilidad al AM, utilizando el fenotipo de Fried ${ }^{7}$.

La fragilidad no está presente en todos los $\mathrm{AM}$, se asocia al envejecimiento pero no es un proceso ineludible a éste, se instala lentamente lo que permite detectarla y prevenirla retardando su presencia, que puede llegar a más de $32 \%$ en los AM de 90 años de edad y causar dependencia ${ }^{11}$.

Así, el objetivo de este estudio es determinar la presencia de los indicadores de fragilidad, en la población chilena de AM a contar de los 65 años ${ }^{12}$, beneficiaria de FONASA y su asociación con el riesgo de pérdida de funcionalidad de acuerdo a la evaluación del EFAM (sección B), estableciendo aquellos factores biopsicosociales que pueden influir tanto en el riesgo de dependencia como en la fragilidad.

\section{Material y Método}

El diseño fue descriptivo correlacional. El universo consideró la población de 65 y más años beneficiarios FONASA, e inscritos en algún Centro de Salud Familiar (CESFAM) de la ciudad, lo que correspondió a 21.099 AM. Se trabajó con muestra aleatoria estratificada con afijación proporcional óptima. El tamaño de los estratos se obtuvo de la Corporación Municipal de Desarrollo Social de la ciudad. El tamaño de muestra calculado con $95 \%$ de confianza y un margen de error en la estimación de la proporción de 0,04 corresponde a $632 \mathrm{AM}$. Se alcanzó una muestra de 754.
Se confeccionó una encuesta que incluyó variables sociodemográficas como: edad, género, ingresos económicos y nivel educacional entre otras. Se incorporaron instrumentos validados para Chile como: el EFAM ${ }^{13}$, y la escala de autoeficacia percibida $^{14}$. Se revisó en el carnet de salud del AM el diagnóstico médico de patologías. Se consultó la existencia de síndromes geriátricos como: incontinencia urinaria, caídas y polifarmacia ${ }^{15}$. Se incluyó cuatro criterios señalados por Fried et $\mathrm{al}^{7}$, como fenotipo de fragilidad: 1) pérdida de peso involuntaria superior a 4,5 kilos del peso previo al último año, se comprobó con carnet de salud; 2) disminución en $20 \%$ de la fuerza de agarre de la mano dominante, medido con un dinamómetro hidráulico (Jamer) ajustado según sexo e IMC; 3) pobre resistencia, definida por autopercepción de fatiga fácil frente a las actividades habituales; 4) actividad física, se aceptó válido el autoreporte de disminución de ésta. La ausencia de estos indicadores corresponde a la categoría "sin fragilidad" la presencia de 1 ó 2 criterios indican "pre-fragilidad" y 3 o más corresponde a "fragilidad"16-18. Previo a la recolección de datos, se realizó una capacitación a enfermeras de atención primaria y estudiantes seminaristas de Enfermería, Universidad de Antofagasta para estandarizar el manejo de las mediciones de la encuesta.

Criterio de inclusión: Mínimo 65 años de edad, adscrito a algún CESFAM. Exclusión: Dependencia, discapacidad física, deterioro cognitivo severo, postrados, y/o analfabetos.

Requerimientos éticos: Se confeccionó el protocolo para el estudio con seres humanos y la carta de consentimiento informado, ambos documentos fueron evaluados y aprobados por el comité de Ética en investigación científica de la Universidad de Antofagasta. En terreno a los AM, se les leía la información que incorporaba riesgos y beneficios del estudio, posteriormente firmaban el consentimiento y se les entregaba una copia con información de contacto de la investigadora responsable.

\section{Técnica de análisis de datos}

Caracterización descriptiva de los resultados, con análisis de asociación, utilizando pruebas $\chi^{2}$. El nivel de medición de las variables en su mayoría corresponde al nivel ordinal o nominal, por lo tanto se utilizó la prueba de Kruskal Wallis para verificar si existen diferencias entre los niveles de 
Fragilidad en adultos mayores en la ciudad de Antofagasta - C. Tapia et al

fragilidad. Los datos fueron procesados con el software SPSS. Versión 20.

\section{Resultados}

Las principales características descriptivas de la muestra fueron: $61,4 \%$ mujeres; el rango de edad osciló entre los 65 y 90 años, con una media de 73 años $(\mathrm{DE}=6,0)$ la distribución porcentual por categoría edad fue: $38,9 \%$ para 75 y más años; $32,9 \%$ para $65-69$ años y $28,2 \%$ los 70 a 74 años. Se declaró casado $40,8 \%$ y viudo $37,3 \%$. La escolaridad se midió por años de estudio, así de 2 a 6 años alcanzó $78,9 \%$. El ingreso económico con mayor representación fue de $\$ 80.000$ a $\$ 152.000$, con $34,6 \%$ (Tabla 1). Las enfermedades crónicas de mayor frecuencia (Tabla 2) fueron: hipertensión arterial $(63,1 \%)$ dislipidemia $(25,9 \%)$ y diabetes mellitus (24,4\%). Un 18,2\% presentaba diabetes e hipertensión arterial; el test Mini-Mental del EFAM clasificó como normal a 95,5\% y alterado a $4,5 \%$ de la muestra. El síndrome geriátrico de mayor frecuencia fue la polifarmacia $(35,9 \%)$,

Tabla 1. Distribución porcentual de características biodemográficas en AM de 65 y más años beneficiarios de FONASA, inscritos en los Centros de Salud Familiar de la ciudad de Antofagasta 2012

\begin{tabular}{|llrr|}
\hline Variables & & $\mathbf{n}$ & \multicolumn{1}{c}{$\%$} \\
& Hombre & 291 & 38,6 \\
& Mujer & 463 & 61,4 \\
Edad (años) & $65-69$ & 248 & 32,9 \\
Categorías & $70-74$ & 213 & 28,2 \\
& 75 y más años & 293 & 38,9 \\
Estado civil & Casado & 308 & 40,8 \\
& Viudo & 281 & 37,3 \\
& Soltero & 82 & 10,9 \\
& Divorciado & 71 & 9,4 \\
Curso alcanzado & Otros & 12 & 1,6 \\
último año lo lee y escribe & 21 & 2,8 \\
(escolaridad) & 2 a 6 años & 595 & 78,9 \\
Nivel de ingresos & 7 y más años & 138 & 18,3 \\
económicos (\$) & 0-79.000 & 72 & 9,5 \\
& 80.000-152.000 & 261 & 34,6 \\
& 153.000-200.000 & 178 & 23,6 \\
& 201.000-300.000 & 115 & 15,3 \\
& 301.000- 400.000 & 60 & 8,0 \\
& Mayor a 400.000 & 57 & 7,6 \\
& No contesta & 11 & 1,5 \\
\hline
\end{tabular}

Fuente: Proyecto FONIS SA11i2179. incontinencia urinaria en la categoría "a veces" $(27 \%)$ y las caídas (26,5\%). Respecto del índice de masa corporal (IMC), la mayor distribución porcentual fue para normalidad con $41,5 \%$ y el sobrepeso $31,1 \%$. En otras características de salud

Tabla 2. Distribución porcentual de antecedentes mórbidos en AM de 65 y más años beneficiarios de FONASA, inscritos en los Centros de Salud Familiar de la ciudad de Antofagasta 2012

\begin{tabular}{|llrc|}
\hline Variables & & n & \% \\
\hline Enfermedades & Hipertensión arterial & 476 & 63,1 \\
Pre-existentes & Diabetes mellitus & 184 & 24,4 \\
& Hipertensión y diabetes & 137 & 18,2 \\
& Dislipidemia & 195 & 25,9 \\
& Artritis/artrosis & 178 & 23,6 \\
& Hipotiroidismo & 90 & 11,9 \\
& LCFAVPOC & 32 & 4,2 \\
Mini mental & Cáncer & 20 & 2,7 \\
EFAM & Alterado & 34 & 4,5 \\
Caídas en el & Normal & 720 & 95,5 \\
último año & Sí & 200 & 26,5 \\
Índice de masa & Enflaquecido & 554 & 73,5 \\
corporal (ajustado & Normal & 94 & 12,5 \\
para AM) & Sobrepeso & 313 & 41,5 \\
& Obesidad & 236 & 31,1 \\
Incontinencia & Nunca & 111 & 14,7 \\
urinaria & A veces & 535 & 71 \\
\hline & Siempre & 202 & 27 \\
& & 17 & 2,0 \\
\hline
\end{tabular}

Fuente: Proyecto FONIS SA11i2179.

Tabla 3. Distribución porcentual de características de salud en AM de 65 y más años beneficiarios de FONASA, inscritos en los Centros de Salud Familiar de la ciudad de Antofagasta 2012

\begin{tabular}{|llcr|}
\hline Variables & & n & \% \\
Autopercepción & Mala & 28 & 3,7 \\
de salud & Regular & 208 & 27,6 \\
& Buena & 366 & 48,5 \\
& Muy buena & 110 & 14,6 \\
& Excelente & 42 & 5,6 \\
Autoeficacia & Baja & 239 & 31,7 \\
& Alta & 515 & 68,3 \\
Apoyo social & Sin apoyo social & 442 & 58,6 \\
& Apoyo social regular & 229 & 30,4 \\
& Apoyo social alto & 83 & 11,0 \\
Polifarmacia canti- & Bajo consumo (0-2) & 483 & 64,1 \\
dad de fármacos & Alto consumo (3 o más) & 271 & 35,9 \\
\hline
\end{tabular}

Fuente: Proyecto FONIS SA11i2179. 
Tabla 4. Distribución de indicadores de fragilidad en AM de 65 y más años beneficiarios de FONASA, inscritos en los Centros de Salud Familiar de la ciudad de Antofagasta 2012

\begin{tabular}{|lcccccc|}
\hline Indicadores de fragilidad & \multicolumn{4}{c}{ Categorías } \\
& $\begin{array}{c}\text { Sin fragilidad } \\
\text { \% }\end{array}$ & $\begin{array}{c}\text { Con fragilidad } \\
\text { \% }\end{array}$ & \multicolumn{2}{c|}{ Total } \\
\hline Dinamometría & 309 & 40,98 & 445 & $\mathbf{5 9 , 0 2}$ & 754 & 100 \\
Autoreporte de disminución de actividad física & 558 & 74,01 & 196 & 25,99 & 754 & 100 \\
\hline Percepción de fatiga & 690 & $\mathbf{9 1 , 5 1}$ & 64 & 8,49 & 754 & 100 \\
\hline Pérdida de peso & 683 & $\mathbf{9 0 , 5 8}$ & 71 & 9,42 & 754 & 100 \\
\hline
\end{tabular}

Fuente: Proyecto FONIS SA11i2179; $p$ value $=0,000 ; \chi^{2} 659,57$

(Tabla 3), 68,3\% calificó con una alta autoeficacia y $58,6 \%$ se declaró sin apoyo social. La autopercepción de salud fue positiva, alcanzó $68,7 \%$ distribuida desde buena hasta excelente.

Entre los indicadores de fragilidad (Tabla 4), la fuerza de agarre, clasificó como frágiles a 59,02\% de la muestra transformándose en el indicador de mayor presencia para la fragilidad, el autoreporte de disminución de la actividad física fue el otro indicador que determinó fragilidad en $25,9 \%$. Tanto la percepción de fatiga $(91,5 \%)$ como la pérdida de peso $(90,5 \%)$ alcanzan mayor distribución porcentual para la categoría sin fragilidad o ausencia de ese criterio. La suma de estos criterios, determinaron niveles de fragilidad los cuales se distribuyeron en $26,5 \%$ sin fragilidad; pre frágil $69,0 \%$ y frágil $4,5 \%$ (Tabla 5).

$\mathrm{Al}$ estudiar la relación, entre la sección $\mathrm{B}$ del EFAM y niveles de fragilidad, esta presentó significancia estadística con un valor de $\mathrm{p}=0,008$. En los AM con riesgo el mayor porcentaje es para la fragilidad $(64,71 \%)$, mientras que para los autovalentes sin riesgo la mayoría corresponde a "Sin fragilidad" (63,5\%). La pre-fragilidad, se distribuye porcentualmente en $59,23 \%$ para los autovalentes sin riesgo, y en $40,77 \%$ con riesgo (Figura 1).

A través de la prueba Kruskal Wallis, se compararon los niveles de fragilidad según las características biodemográficas como: edad categorizada, sexo, último curso de educación formal y características de estado de salud como: autopercepción de salud, IMC, puntajes de la escala de autoeficacia y del Mini-Mental; como en todas las categorías de estas variables, la diferencia observada entre los tres rangos promedios fue estadísticamente significativa, se puede plantear que la distribución de los puntajes en los niveles de fragilidad es diferente para estas variables (Tablas 6 y 7 ).

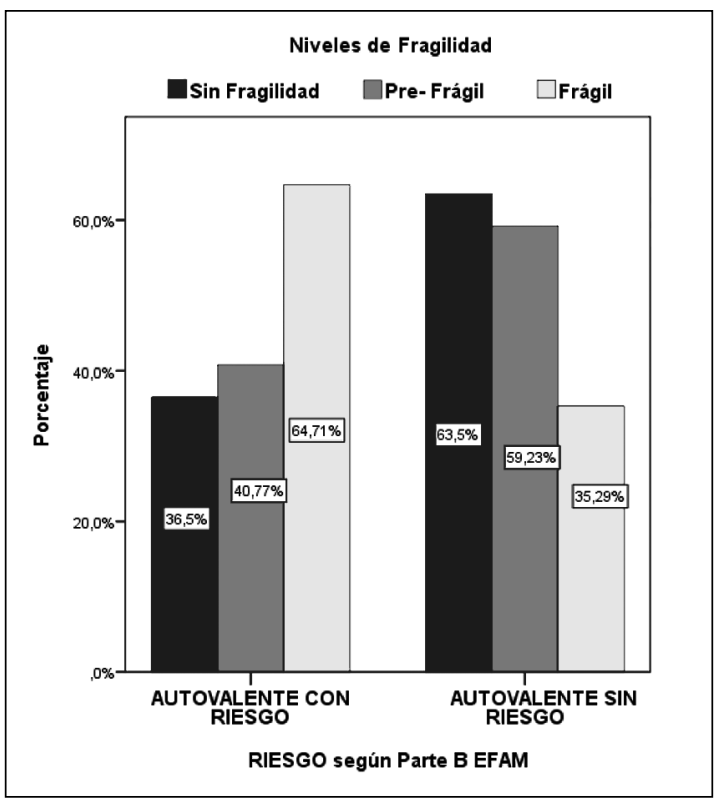

Figura 1. Distribución porcentual entre los niveles de fragilidad y categorías de la sección B del EFAM, AM de 65 y más años, beneficiarios de FONASA, inscritos en los Centros de Salud Familiar de la ciudad de Antofagasta 2012. Fuente: Proyecto FONIS SA11/2179.

Tabla 5. Distribución de niveles de Fragilidad en AM de 65 y más años beneficiarios de FONASA, inscritos en los Centros de Salud Familiar de la ciudad de Antofagasta 2012

\begin{tabular}{|c|c|c|}
\hline Niveles de fragilidad & Frecuencia & $\%$ \\
\hline Sin fragilidad (0 criterios) & 200 & 26,5 \\
\hline ( $1-2$ criterios) & 520 & 69,0 \\
\hline (3-4 criterios) & 34 & 4,5 \\
\hline Total & 754 & 100,0 \\
\hline
\end{tabular}

Fuente: Proyecto FONIS SA11i2179. 
Fragilidad en adultos mayores en la ciudad de Antofagasta - C. Tapia et al

Tabla 6. Prueba de Kruskal-Wallis. Niveles de fragilidad y características biodemográficas en AM de 65 y más años beneficiarios de FONASA, inscritos en los Centros de Salud Familiar de la ciudad de Antofagasta 2012

\begin{tabular}{|llcc|}
\hline Rangos & & & \\
Variables & Niveles de fragilidad & n & Rango promedio \\
\hline Edad en categorías & Sin fragilidad & 200 & 323,16 \\
& Pre frágil & 520 & 397,49 \\
& Frágil & 34 & 391,38 \\
\hline Sexo & Sin fragilidad & 200 & 385,40 \\
& Pre frágil & 520 & 365,98 \\
Cuso alcanzado en educación formal & Srágil fragilidad & 34 & 414,97 \\
(escolaridad) & Pre frágil & 200 & 416,82 \\
& Frágil & 520 & 364,70 \\
\hline
\end{tabular}

Fuente: Proyecto FONIS SA11i2179. U. A.

Estadístico de contraste

\begin{tabular}{|l|c|c|c|}
\hline & Edad en categorías & Sexo & Último curso en educación formal \\
\hline$\chi^{2}$ & 19,216 & 9,668 & 9,532 \\
\hline gl & 2 & 2 & 2 \\
\hline Sig. asintót. & 0,000 & 0,008 & 0,009 \\
\hline
\end{tabular}

Tabla 7. Prueba de Kruskal-Wallis. Niveles de fragilidad y características del estado de salud en AM de 65 y más años beneficiarios de FONASA, inscritos en los Centros de Salud Familiar de la ciudad de Antofagasta 2012

\begin{tabular}{|llcc|}
\hline $\begin{array}{l}\text { Rangos } \\
\text { Variables }\end{array}$ & Niveles de fragilidad & n & Rango promedio \\
\hline Índice de masa corporal & Sin fragilidad & 200 & 335,87 \\
& Pre frágil & 520 & 392.04 \\
\hline Autopercepción de salud & Frágil & 34 & 400,03 \\
& Sin fragilidad & 200 & 401,62 \\
Puntaje minimental & Frágil & 520 & 375,12 \\
& Sin fragilidad & 34 & 272,00 \\
\hline Puntaje escala autoeficacia & Pre frágil & 200 & 416,96 \\
& Frágil & 520 & 363,77 \\
& Sin fragilidad & 34 & 355,41 \\
\hline & Pre frágil & 200 & 392,03 \\
\hline
\end{tabular}

Fuente: Proyecto FONIS SA11i2179. U. A.

Estadístico de contraste

\begin{tabular}{|l|c|c|c|c|}
\hline & Autopercepción de salud & Puntaje del Mini mental & Puntaje escala de autoeficacia & Índice de masa corporal \\
\hline$\chi^{2}$ & 12,181 & 9,672 & 9,902 & 11,187 \\
\hline gl & 2 & 2 & 2 & 2 \\
\hline Sig. asintót. & 0,002 & 0,008 & 0,007 & 0,004 \\
\hline
\end{tabular}




\section{Tabla 8.Tabla de correlaciones. Niveles de fragilidad según hipertensión arterial/diabetes mellitus e hipertensión arterial/dislipidemia en AM de 65 y más años beneficiarios de FONASA, inscritos en los Centros de Salud Familiar de la ciudad de Antofagasta 2012}

\begin{tabular}{|c|c|c|c|c|c|}
\hline \multicolumn{6}{|c|}{ Correlaciones } \\
\hline \multirow{4}{*}{$\begin{array}{l}\text { Rho de } \\
\text { Spearman }\end{array}$} & & & $\begin{array}{l}\text { Nivel de } \\
\text { fragilidad }\end{array}$ & $\begin{array}{l}\text { Hipertensión } \\
\text { con dislipidemia }\end{array}$ & $\begin{array}{l}\text { Hipertensión con } \\
\text { diabetes mellitus }\end{array}$ \\
\hline & Nivel de fragilidad & $\begin{array}{l}\text { Coeficiente de correlación } \\
\text { Sig. (bilateral) } \\
n\end{array}$ & $\begin{array}{r}1,000 \\
754\end{array}$ & $\begin{array}{l}, 025 \\
, 498 \\
754\end{array}$ & $\begin{array}{l}, 104 * * \\
, 004 \\
754\end{array}$ \\
\hline & $\begin{array}{l}\text { Hipertensión con } \\
\text { dislipidemia }\end{array}$ & $\begin{array}{l}\text { Coeficiente de correlación } \\
\text { Sig. (bilateral) } \\
n\end{array}$ & $\begin{array}{l}, 025 \\
498 \\
754\end{array}$ & $\begin{array}{r}1,000 \\
754\end{array}$ & $\begin{array}{l}, 697 * * \\
, 000 \\
754\end{array}$ \\
\hline & $\begin{array}{l}\text { Hipertensión con } \\
\text { diabetes mellitus }\end{array}$ & $\begin{array}{l}\text { Coeficiente de correlación } \\
\text { Sig. (bilateral) } \\
n\end{array}$ & $\begin{array}{l}, 104 * * \\
, 004 \\
754\end{array}$ & $\begin{array}{l}697 * * \\
, 000 \\
754\end{array}$ & $\begin{array}{r}1,000 \\
754\end{array}$ \\
\hline
\end{tabular}

Fuente: Proyecto FONIS SA11i2179. ** La correlación es significativa al nivel 0,01 (bilateral).

Para analizar las enfermedades pre-existentes, se seleccionó las tres patologías que alcanzaron la mayor distribución y se correlacionó cada una de estas con el nivel de fragilidad, utilizando el estadístico de contraste Rho de Spearman. Los resultados fueron positivos para la diabetes mellitus, sola o asociada con hipertensión arterial, se puede plantear que el nivel de fragilidad se asoció con la presencia de la diabetes sola o en conjunto con la hipertensión arterial según el valor de p. Se muestra el resultado sólo con las patologías asociadas (Tabla 8).

\section{Discusión}

Los datos descriptivos enfatizan el aumento porcentual de AM sobre los 70 años, así, en la misma ciudad el año $2009^{19}$ este rango etario alcanzó $43,9 \%$, versus $67,1 \%$ del estudio realizado el 2013. En esta población más envejecida aumenta la prevalencia de dependencia ${ }^{3}$ y se acompaña de fragilidad. El criterio "fuerza de agarre" ${ }^{20}$ corresponde al más utilizado para establecer fragilidad, determinando la mayoría porcentual $(59,2 \%)$, entre los otros criterios.

La dinamometría de mano dominante, tanto a nivel internacional ${ }^{21}$ como nacional $^{22}$ se asocian con funcionalidad y la capacidad de realizar actividades que involucran movimiento, en consecuencia es el segundo criterio que estableció fragilidad en $25,99 \%$ evaluado como la disminución de actividad física. Al comparar los niveles de fragilidad con otro estudio realizado en una realidad socioeconómica similar ${ }^{17}$, existen leves diferencias mientras $69,0 \%$ de los AM antofagastinos resultan pre frágiles, y 4,5\% frágil, en Lima la pre-fragilidad es menor $(64,6 \%)$ y la fragilidad mayor $(7,7 \%)$. Estas pueden explicarse por diferencias en el tipo, tamaño muestral y cómo se midió los criterios de Fried, sin embargo, en ambas ciudades a mayor edad aumentó la asociación con fragilidad, lo que se ha demostrado en estudios iberoamericanos y europeos ${ }^{20,23}$.

Se observaron diferencias estadísticamente significativas que avalan que la fragilidad es diferente según algunos factores biodemográficas y psicosociales ${ }^{7,16,17}$. Así, a mayor edad y ser mujer se asoció con mayor riesgo de fragilidad, teóricamente se esperó este resultado por el alto porcentaje de mujeres $(61,4 \%)$ lo que concuerda con otras investigaciones ${ }^{12,20}$.

Campbell et $\mathrm{al}^{5}$, incorporaron la evaluación cognitiva como factor psicosocial componente de fragilidad, posteriormente L. Fried et $\mathrm{al}^{7}$, demostró asociación entre el Mini-Mental, el nivel de escolaridad y la autopercepción de salud con la fragilidad. Se obtuvo resultados similares en estas variables; sin embargo, el nivel de ingresos y el apoyo social no alcanzó asociación con la fragilidad, lo que puede ser consecuencia del tamaño muestral, diseño de la investigación y/o como se midieron estas variables. 
Las enfermedades crónicas concuerdan con la encuesta de salud ${ }^{23}$ así la mayor prevalencia, se encontró para: hipertensión arterial $(63,1 \%)$, dislipidemia $(25,9 \%)$ diabetes mellitus $(24,4 \%)$ y artritis/artrosis (23,6\%), la comorbilidad también se señala como probable causa y/o predictor de fragilidad $^{7,8,24,25}$, en este estudio se demostró que el nivel de fragilidad se correlacionó al nivel 0,01 bilateral con la Diabetes Mellitus sola o con la hipertensión arterial. A través de la prueba de Kruskal Wallis se demostró que el nivel de fragilidad es diferente según el IMC, el cual, determinó 31,1\% de sobrepeso y $14,7 \%$; de obesidad, cifras más bajas que las declaradas en el artículo específico para este grupo etario ${ }^{22}$ como en la ENS 2009-201023. Es importante destacar, que la obesidad es un predictor tanto de discapacidad como de fragilidad ${ }^{26}$.

Se constató relación con significancia estadística $(\mathrm{p} \leq, 008)$ entre el EFAM, sección $\mathrm{B}$ y la fragilidad. En los AM autovalentes sin riesgo se encontró $63,5 \%$ sin fragilidad, 59,23\% de pre-fragilidad y $35,29 \%$ de fragilidad. El AM autovalente con riesgo ${ }^{13}$, es derivado a médico y/o educación en el programa cardiovascular que corresponda; si es autovalente sin riesgos debe ser derivado a acciones de promoción y prevención. Esta indicación descansa en la facilidad que el entorno comunitario le ofrece al $\mathrm{AM}^{27}$, porque en salud las acciones están más orientadas a la rehabilitación ${ }^{28}$. Son autovalentes sin riesgo pero presentan criterios de fragilidad, lo que los deja con mayor vulnerabilidad frente a eventos adversos de cualquier origen.

Considerando que el EFAM incorpora algunas mediciones señaladas como criterios de fragili$\operatorname{dad}^{5,7,23,29}$ se propone incorporar una medición objetiva de fragilidad, la dinamometría capaz de discriminar riesgo de fragilidad siguiendo los criterios de Fried, es una medida fácil de implementar en atención primaria. Además de pesquisar pérdida de funcionalidad a través del EFAM, se realizaría un tamizaje básico para fragilidad a la población más envejecida que va en aumento en el país.

\section{Referencias}

1. Instituto nacional de Estadística de Chile, CENSO 2012, Disponible en: http://estudios.anda.cl/recursos/ censo_2012.pdf [Consultado el 30 de julio de 2013].

2. Instituto Nacional de Estadística de Chile. Enfoque Es- tadístico. Adulto Mayor en Chile. Septiembre 2007: 2-4. Disponible en: http://www.ine.cl/canales/sala_prensa/ noticias/2007/septiembre/boletin/ine_adulto_mayor. pdf [Consultado el 05 de junio de 2013].

3. Estudio Nacional de la Dependencia en las Personas Mayores. Disponible en: www.senama.cl/filesapp/Estudio Nacional_dependencia.pdf [Consultado el 30 de enero 2014].

4. Rodríguez L, Castro M. Envejecimiento y Fragilidad. En Guillén F, Pérez del Molino J, Petidier R. Síndromes y Cuidados en el Paciente geriátrico. 2 ed. Barcelona. España. Elsevier Masson; 2008: 21-30.

5. Campbell J, Buchner D. Unstable disability and the fluctuations of frailty. Age and Aging, 1997; 26: 315-8.

6. Montero M, Muir S, Hall M, Doherty T, Kloseck M, Beauchet $\mathrm{O}$, et al. Gait Variability Is Associated With Frailty in Community-dwelling Older Adults. Journal of Gerontology. Serie A.2011; 66 (5): 568-76.

7. Fried L, Tangen C, Walson J, Newman A, Hirsch C, Gottdiener J, et al. Frailty in older adults: Evidence for a phenotype. J Gerontology Series A-Bio Sci \& Med Sci 2001; 56(3): 146-56.

8. Superintendencia de Salud de Chile. Análisis estadístico del sistema Isapre con enfoque de Género 2011. Disponible en: http://www.supersalud.gob.cl/documentacion/569/articles-8203_recurso_1.pdf [Consultado el 29 de julio de 2013].

9. Superintendencia de Salud. Informe final evaluación funcional del adulto mayor. Disponible en www.supersalud.gob.cl/documentacion/569/w3-article-4393 [Consultado el 30 de enero de 2014].

10. Ministerio de Salud de Chile. Subsecretaria de Salud Pública. Guía Clínica; Adulto Mayor Frágil. Septiembre 2008. Disponible en: Http://Www.Ssmaule.Cl/ Paginas/Index2.Php?Option=Com_Docman\&Task=Doc_View\&Gid=2142\&Itemid=96 [Consultado el 27 de abril de 2011].

11. Ahmed N, Mandel R, Fain M. Frailty: An Emerging Geriatric Syndrome. Am J Med 2007; 120 (9): 748-5.

12. Abizanda P, Gómez-Pavón J, Martín I, Baztán J. Detección y prevención de la fragilidad: una nueva perspectiva de prevención de la dependencia en las personas mayores. Medicina Clínica 2010; 135 (15): 713-9.

13. Ministerio de Salud de Chile. Manual de Aplicación del Examen de Medicina Preventiva del Adulto 2012; 4-16. Disponible en http://www.minsal.cl/portal/url/item/ ab1f81f43ef0c2a6e04001011e011907.pdf [Consultado el 27 de abril de 2011]

14. Cid P, Orellana A, Barriga O. Validación de la escala de autoeficacia general en Chile. Rev Med Chile [revista en la Internet] 2010; 138 (5): 551-7. 
15. Organización Panamericana de la Salud 2010: 209-222. Disponible en: http://www.paho.org/blogs/paltex/ wp-content/uploads/2013/06/saluddelosadultosmayores_preliminares.pdf?ua $=[$ Consultado el 12 de marzo de 2015].

16. Levers M, Estabrooks C, Ross J. Factors contributing to frailty: literature review. J Adv Nurs 2006; 56 (3): 28291.

17. Varela-Pinedo L, Ortiz-Saavedra P, Chávez-Jimeno H. Síndrome de Fragilidad en adultos mayores de la comunidad de Lima Metropolitana. Rev Soc Peru Med Interna 2008; 21 (1): 11-5.

18. Rockwood K. Frailty and its definition: a worthy challenge. J Am Geriatr Soc 2005; 53 (6): 1069.

19. Tapia C, Varela H, Barra L, Ubilla M, Iturra V, Collao C, et al. Valoración multidimensional del envejecimiento en la ciudad de Antofagasta. Rev Med Chile 2010; 138: 444-51.

20. Macklai N, Spagnoli J, Junod J, Santos-Eggimann B. Prospective association of the SHARE-operationalized frailty phenotype with adverse health outcomes: evidence from $60+$ community-dwelling Europeans living in 11 countries. BMC Geriatrics 2013; 13 (3): 1-9.

21. Ramlagan S, Peltzer K, Phaswana N. Hand grip strength and associated factors in non-institutionalised men and women 50 years and older in South Africa. BMC. Research Notes 2014; 7: 8.

22. Arroyo P, Lera L, Sánchez H, Bunout D, Santos J, Albala C. Indicadores Antropométricos, composición corporal y limitaciones funcionales en ancianos. Rev Med Chile 2007; 135: 846-54.

23. MINSAL, Superintendencia de Salud, Gobierno de Chile. Encuesta de salud en población general de Chile 2009-2010. Departamento de Estudios del Desarrollo 2010. Disponible en http://www.minsal.gob.cl/portal/ url/item/bcb03d7bc28b64dfe040010165012d23.pdf [Consultado el 14 de junio de 2013].

24. Clegg A, Young J, Iliffe S, Olde M, Rockwood K. Frailty in elderly people. Lancet 2013; 381 (3): 752-62.

25. Jiménez E, Írsula L. Adulto Mayor en estado de fragilidad. MEDISAN [revista en la Internet] 2010; 14 (3): 1-6. Disponible en: http://scielo.sld.cu/scielo.php?scrip$\mathrm{t}=$ sci_arttext\&pid=S1029-30192010000300017\&lng=es [Consultado el 14 de junio de 2013].

26. García-García J, Larrión JL, Rodríguez L. Fragilidad: un fenotipo en revisión. Gac Sanit 2011; 25 (5): 51-8.

27. Annear M, Keeling S, Wilkinson T, Cushman G, Gidlow B, Hopkins H. Environmental influences on healthy and active ageing: a systematic review. Ageing and Society 2013; 3: 1-33.

28. Estrategia Nacional de Salud 2011-2020. Disponible en: http://gob.cl/especiales/esttrategia-nacional-de salud-2011-2010 [Consultado el 10 de febrero de 2014].

29. Malaguarnera M, Vacante M, Frazzetto P, Massimo $M$. What is the frailty in ederly? Value and significance of the multidimensional assessments. Arch Gerontol Geriatr 2013; 56: 23-6. 